Cuidador:

\title{
¿concepto operativo o preludio teórico?
}

\section{Caregiver: operative concept or theoretical prelude?}

Mtro. José Cruz Rivas Herrera.

Mtra. Rosa Ma. Ostiguín Meléndez••

\section{Resumen}

Introducción: El concepto cuidador desde los 70`s ha tomado sin duda un auge particular, sin embargo hoy por hoy existe una diversidad de términos que frecuentemente se emplean como sinónimos, pero que desde el particular punto de vista tienen implicaciones teóricas mucho más profundas. Desarrollo: El presente artículo expone algunas consideraciones en torno al concepto de cuidador que se identificó en la literatura en español con instrumentos relacionados a esta figura en el periodo 2008-2009; particularmente se recupera el quién cuida?, qué hace?, qué relación guarda con la persona que cuida?, entre otros; todo ello, para constituirse en un referente al respecto del término. Conclusiones: Los hallazgos más relevantes apuntan a que el termino cuidador adquiere dimensiones varias en la medida que se identifica el vinculo de la persona que ofrece el cuidado y las acciones están centradas en los conceptos de apoyo o ayuda.

\section{Abstract}

Intruduction: The concept caretaker from the $70^{\prime}$ s, has taken a particular peak without a doubt, however today per today it exists a diversity of terms that frequently are used as synonyms, but that from the particular point of view they have much deeper theoretical implications. Development: The present article exposes some considerations around caretaker's concept that was identified in the literature in Spanish with instruments related to this figure in the period 2008-2009; particularly recover the who takes care?, how ago? what relationship keep with the person that takes care?, among others; everything it, to be constituted in a with respect to the one regarding the term. Conclusions: The most excellent discoveries point to that the one finishes caretaker acquires several dimensions in the measure that is identified the link of the person that offers the care and the actions are centered in the support concepts or helps.
Palabras

Clave:

Cuidador, cuidador familiar, cuidador informal, cuidador primario.

- Mtro. en Enfermería, Prof. Asig. ENEO-UNAM.

•. Mtra. en Enfermería, Coordinadora del Programa de Maestría en Enfermería. UNAM.

Correspondencia: joserivash@gmail.com

RECIBIDO: 27 DE SEPTIEMBRE DE 2010.

ENVIADO: 16 DE OCTUBRE DE 2010.

ACEPTADO:10 DE DICIEMBRE DE 2010. 


\section{INTRODUCCIÓN}

En México al hablar de cuidador con frecuencia provoca pensar en el estrés del familiar de un enfermo quien dedica su tiempo a servirlo, sin embargo la revisión de la literatura de artículos en español y con instrumentos sobre cuidadores de personas adultas con enfermedades crónicas tales como cáncer, Alzheimer, insuficiencia renal, por mencionar algunas, permitió identificar que existen una serie de términos que se utilizan como sinónimos, para referir al sujeto de interés: el cuidador, en la investigación de enfermería.

La dificultad de esta situación sería mínima, si se partiera de que los conceptos hacen el escenario sobre el cual el investigador reconoce diversos ángulos del sujetoobjeto de estudio, pero cuando son restringidos a una dimensión semántica, se presume una limitante para el iniciado en la investigación.

De esta manera el presente articulo pretende ofrecer al lector la posibilidad de reconocer algunos elementos tales como: quién cuida?, que relación guarda con la persona que cuida?, que tareas hace para cuidar? y el nivel de participación; como elementos que permitan discutir al respecto del cuidador latino como recurso para la salud, dimensionar a la familia y con ello vislumbrar la competencia del profesional de la enfermería ante esta figura.

\section{Cuidador: algunas CONSIDERACIONES}

El tema del cuidador ha sido multiestudiado, existe evidencia de su abordaje desde el perfil, ${ }^{3,4,5}$ el desgaste que sufre ${ }^{6,7,8}$, las habilidades que desarrolla, $, 910,11$ etc. $y$ en todos
Actualmente se está produciendo un cambio en los modelos familiares de cuidado, dados por los perfiles epidemiológicos contemporáneos,

los casos se vincula en mayor o menor grado con la familia., ${ }^{7,12,13}$

La relevancia de explorar el término de cuidador está justamente en reconocer su naturaleza y su participación directa en el cuidado de enfermos crónicos, ${ }^{7,14,15}$ así como su carácter instrumental en la recuperación, ${ }^{4,16}$ bienestar o restablecimiento de personas enfermas, generalmente de larga duración.

Actualmente se está produciendo un cambio en los modelos familiares de cuidado, dados por los perfiles epidemiológicos contemporáneos, en donde las enfermedades que ayer eran conceptualizadas como agudas y de muerte inminente al día de hoy son crónicas y de cuestionable calidad de vida y, con ello un proceso de cuidado ampliado al hogar, a la familia y por supuesto a un cuidador del mismo núcleo.

Esta situación se ha traducido para los integrantes de cada familia en la designación o asunción de la labor de cuidador de la persona dependiente en el núcleo familiar. ${ }^{17,18,19}$ Estos cambios de la asistencia del individuo en su domicilio y la aparición de figuras como agentes de su salud, cuidadores familiares, entre otros hacen que la dinámica familiar sea una variable muy importante.

Proporcionar cuidados a un enfermo precisa de apoyo, por- que atenderlo en casa representa un cambio sustancial para la familia por tiempo prolongado, que produce con frecuencia una serie de síntomas que desgastan en lo físico, emocional, económico, etc. ${ }^{20,21}$ La labor del cuidador tiene una gran relevancia, no sólo por la atención directa al paciente, sino también por su papel en la reorganización, mantenimiento y cohesión de la familia, ${ }^{22}$ superación de la vulnerabilidad de la enfermedad, ${ }^{23}$ entre otras.

Bajo este contexto y a partir del análisis de la literatura en español, se identificó que la utilización del término va desde aquel individuo que funge como acompañante de la persona que se cuida, ${ }^{24}$ hasta el que toma decisiones sobre el cuidado de otro. ${ }^{10,25}$

\section{Concepciones del Cuidador}

Considerando lo dicho líneas arriba al respecto del cuidador, es innegable que la familia es el escenario donde se desarrolla y conserva el cuidado y es justamente desde su interior que emerge la figura del cuidador.

El cuidador es en no pocas ocasiones el recurso, instrumento y medio por el cual se proveen cuidados específicos y muchas veces especializados a los enfermos crónicos, es decir, en ellos se deposita o descansa el compromiso de preservar la vida de otro. Así y con esa responsabilidad en hombros, vale la pena identificar la nomenclatura que al respecto existe.

Inicialmente en la revisión de 20 artículos, se identificaron 7 conceptos asociados al término de cuidador, dos compuestos y cinco simples; mismos que se analizan en función de la concepción operativa 
que se ofrece en los reportes de investigación (Figura 1).

En estos conceptos, se reconoce en principio que todos son referentes operativos de las investigaciones que les dieron origen, sin un marco teórico explícito al respecto, si partimos del significado de esto, es tanto como decir que los instrumentos generados de estas investigaciones tienen sentido aplicativo dada su naturaleza operativa, solo para el investigador en su circunstancia y no en otra.

Por otro lado los conceptos, identificados en los cuadros 1a y $1 b$, exponen al menos cuatro elementos sustantivos sobre los cuales se construye el concepto de cuidador: el quién cuida?, qué hace?, nivel de participación, lugar del

\section{Figura 1}

\section{Conceptos asociados al cuidador}

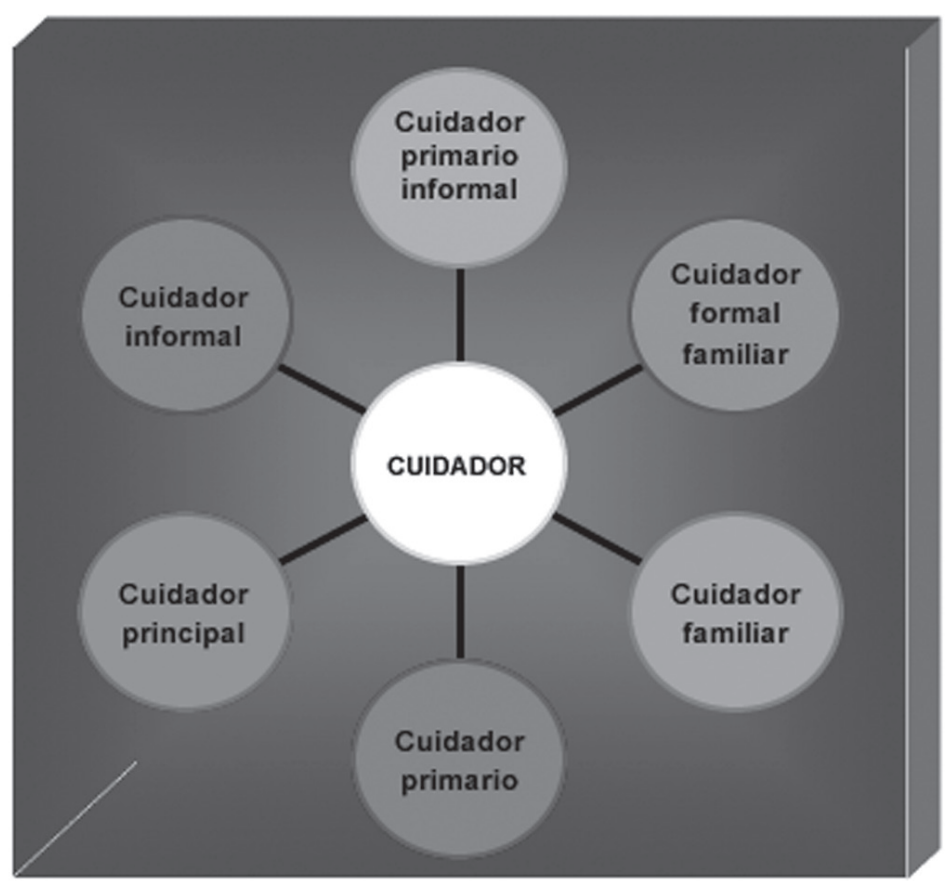

\section{Cuadro 1a}

\section{Conceptos operativos del término cuidador identificados en la literatura}

\begin{tabular}{|c|c|c|}
\hline TERMINO & CONCEPTO & INSTRUMENTO \\
\hline 1 Cuidador & $\begin{array}{l}\text { Aquella persona que asiste o cuida a otra afectada de cual- } \\
\text { quier tipo de discapacidad, minusvalía o incapacidad que } \\
\text { le dificulta o impide el desarrollo normal de sus actividades } \\
\text { vitales o de sus relaciones sociales. }{ }^{24}\end{array}$ & $\begin{array}{l}\text { Construyendo el concepto } \\
\text { cuidador de ancianos } \\
\text { (Reyes, 2001) }\end{array}$ \\
\hline Cuidador & $\begin{array}{l}\text { Es un concepto mixto formado en gran medida por la reali- } \\
\text { zación de tareas de carácter de apoyo, pero definido también } \\
\text { en términos de relaciones sociales y familiares, generalmente } \\
\text { parentesco, aunque se extiende a lazos de amistad o de ve- } \\
\text { cindad donde éstos han adquirido el carácter de relaciones } \\
\text { primarias. }^{26}\end{array}$ & $\begin{array}{l}\text { Grupo de cuidador prima- } \\
\text { rio de daño cerebral: } \\
\text { una perspectiva de análisis } \\
\text { (Bergero, 2000) }\end{array}$ \\
\hline $\begin{array}{l}\text { 2. Cuidador } \\
\text { principal }\end{array}$ & $\begin{array}{l}\text { Es la persona familiar o cercana que se ocupa de brindar de } \\
\text { forma prioritaria apoyo tanto físico como emocional a otro } \\
\text { de manera permanente y comprometida }{ }^{10} \text {. }\end{array}$ & $\begin{array}{l}\text { Habilidad del cuidador y } \\
\text { funcionalidad de la persona } \\
\text { cuidada (Venegas, 2006) }\end{array}$ \\
\hline $\begin{array}{l}\text { Cuidador } \\
\text { principal }\end{array}$ & $\begin{array}{l}\text { El término se utilizaba para referirse a los miembros de la } \\
\text { familia u otros importantes que atendieron al paciente en su } \\
\text { domicilio y que fue identificado por el paciente como su cui- } \\
\text { dador principal2 }{ }^{27} \text {. }\end{array}$ & $\begin{array}{l}\text { Manejo del dolor en pacien- } \\
\text { tes con cáncer que reciben } \\
\text { atención domiciliaria } \\
\text { (Vallerend, 2007) }\end{array}$ \\
\hline $\begin{array}{l}\text { 3. Cuidador } \\
\text { informal }\end{array}$ & $\begin{array}{l}\text { Son las personas que no pertenecen a ninguna institución } \\
\text { sanitaria ni social y que cuidan a personas no autónomas que } \\
\text { viven en su domicilio }{ }^{4} \text {. }\end{array}$ & $\begin{array}{l}\text { Cuidadoras informales } \\
\text { en el medio hospitalario } \\
\text { (Celma, 2001) }\end{array}$ \\
\hline
\end{tabular}




\section{Cuadro 1b}

\section{Conceptos operativos del término cuidador identificados en la literatura}

\begin{tabular}{|c|c|c|}
\hline $\begin{array}{l}\text { 4. Cuidador } \\
\text { familiar }\end{array}$ & $\begin{array}{l}\text { Las personas que presten atención a las personas que necesitan } \\
\text { supervisión o asistencia en la enfermedad o la discapacidad. } \\
\text { Ellos pueden proporcionar el cuidado en el hogar, en un hos- } \\
\text { pital o en una institución, el concepto se refiere también a los } \\
\text { padres, cónyuges u otros familiares, amigos, miembros del } \\
\text { clero, profesores, trabajadores sociales, los demás pacientes, } \\
\text { etc. } 28\end{array}$ & $\begin{array}{l}\text { Experiencias del cuida- } \\
\text { dor familiar en el cuida- } \\
\text { do de un paciente con } \\
\text { cáncer (Maeyama, 2007) }\end{array}$ \\
\hline $\begin{array}{l}\text { 5. Cuidador } \\
\text { primario }\end{array}$ & $\begin{array}{l}\text { Es el que pone en marcha la solidaridad con el que sufre y el que } \\
\text { más pronto comprende que no puede quedarse con los bra- } \\
\text { zos cruzados ante la dura realidad que afecta a su familiar o } \\
\text { amigo. }^{29}\end{array}$ & $\begin{array}{l}\text { La calidad de vida en los } \\
\text { cuidadores primarios } \\
\text { de pacientes con cáncer } \\
\text { (Expósito, 2008) }\end{array}$ \\
\hline $\begin{array}{l}\text { Cuidador } \\
\text { primario }\end{array}$ & $\begin{array}{l}\text { Persona que atiende en primera instancia las necesidades físicas } \\
\text { y emocionales de un enfermo: papel que por lo general lo jue- } \\
\text { gan el/la esposo/a, hijo/a, un familiar cercano o alguien que } \\
\text { es significativo para el paciente. }^{30}\end{array}$ & $\begin{array}{l}\text { Desgaste físico y emo- } \\
\text { cional del cuidador pri- } \\
\text { mario en cáncer } \\
\text { (Alvarado, 2009) }\end{array}$ \\
\hline $\begin{array}{l}\text { 6. Cuidador } \\
\text { primario } \\
\text { informal }\end{array}$ & $\begin{array}{l}\text { Asume la responsabilidad total del paciente ayudándole a rea- } \\
\text { lizar todas las actividades que no puede llevar a cabo; gene- } \\
\text { ralmente es un miembro de la red social inmediata (familiar, } \\
\text { amigo o incluso vecino), que no recibe ayuda económica ni } \\
\text { capacitación previa para la atención del paciente. }{ }^{31}\end{array}$ & $\begin{array}{l}\text { Perfil psicosocial del } \\
\text { cuidador primario in- } \\
\text { formal del paciente con } \\
\text { EPOC (Salas, 2006) }\end{array}$ \\
\hline $\begin{array}{l}\text { 7. Cuidador } \\
\text { principal } \\
\text { familiar }\end{array}$ & $\begin{array}{l}\text { Se identifica como aquel cuidador principal familiar reconocido } \\
\text { como primordial agente que da asistencia básica a los pacien- } \\
\text { tes en su condición de enfermedad crónica. Es el apoyo con- } \\
\text { tinuo que lo asiste en lo cotidiano y quien asume las principa- } \\
\text { les decisiones sobre su cuidado. }{ }^{32}\end{array}$ & $\begin{array}{l}\text { El cuidado de los indi- } \\
\text { viduos y de los grupos: } \\
\text { quien se cuida } \\
\text { (Izquierdo, 2009) }\end{array}$ \\
\hline
\end{tabular}

cuidado, vinculo con la persona cuidada.

En los conceptos recuperados en los cuadros 1a y $1 \mathrm{~b}$, se identifica que en cuanto al quien, el concepto de cuidador se centra en tres perfiles:

La persona, que se refiere a cualquiera que apelando a su naturaleza y esencia ${ }^{33}$ actúa.

Familiares con parentesco, es decir aquellos consanguíneos que cumplen la tarea.

Miembro de la red social inmediata (amigos, vecinos, clérigos, etc.)

Sin embargo, el atributo más relevante y común en todas las concepciones operativas es el hecho de que el cuidador es una figura significativa para la persona que será cuidada.

En lo referente al qué, es decir la tarea que le da sentido al cuidador está en función de:
a) Cuidado
b) Asistencia
c) Tareas de apoyo
d) Físico y emocional
c) Prestar atención a las necesidades físicas

La acepción de cuidado aún es imprecisa para la propia disciplina, sin embargo suele tener una connotación de precaución, de esmero en la acción; donde la razón y la emoción vulneran la conciencia del sujeto de estudio, mientras que la asistencia se asocia a términos como ayuda, socorro y amparo, en el cual el pensamiento invita a tener en mente que es necesario contar con otro actor que se presume en desamparo y vulnerabilidad.

Por otro lado, a la atención le subyace un concepto de especial 
cuidado para realizar algo, es un interés esmerado con respecto a algo consecuente con un "estado psíquico que permite orientar y concentrar la actividad cognoscitiva $y$ práctica del hombre en un determinado objeto o en una determinada acción"34 determinada por un objetivo del que se tiene conciencia.

En cuanto al apoyo, el significado se centra en la idea de sostener, proteger y auxiliar a otro.

Bajo estas concepciones primarias es posible inferir el amplio y ambiguo espacio sobre el cual transita la tarea del cuidador y la complejidad de su participación en la relación con otra persona considerada vulnerable y la necesidad de una construcción teórica al respecto.

Paralelamente las tareas del cuidado al parecer se centran en tres acciones: las responsabilidades del cuidado mismo, participar en la toma de decisiones conjuntas a la persona cuidada y poner en marcha la solidaridad con el que sufre.

Del mismo modo para que exista el cuidador se reconoce que debe existir una persona motivo de cuidado con atributos tales como: no autónoma, en condición de cronicidad y discapacidad, enferma, que requiere supervisión; es decir, dependiente.

Con lo expresado es posible inferir que para hablar del cuidador es necesario considerar el contexto donde éste se significa, ya que es en esa acepción donde se identifica el quién, qué y cómo de las acciones especificas que lo distinguen como tal.

A manera de conclusión, podría decirse que el fenómeno del cuidador tiene una multiplicidad de factores sobre los cuales construirse de manera teórica, implica una connotación más profunda, ya que existen una serie de premisas empíricas que le están ofreciendo sustento, sin embargo aun son primarias (Figura 2), por ello la necesidad de seguir analizando los elementos sustantivos que circunscriben el concepto de cuidador más allá del nivel de intervención, la relación con el sujeto de cuidado (familiar o no), la participación en el cuidado (principal o no) o por el hecho de "carecer" de habilidades reconocidas desde el ámbito de una profesión (informal).

En el marco de lo anterior, es posible explicar de alguna manera la diversidad de términos que se hilvanan en torno al cuidador, cada uno tan válido como escenarios y circunstancias existan, sin embargo, es necesario seguir incursionando en experiencias investigativas que permitan teorizar al respecto del cuidador, para con ello ofrecer un anclaje conceptual sobre el cual realizar análisis sobre el fenómeno de interés.

\section{ReferenCIAS BIBLIOGRÁFICAS}

1 Nolan M. Positive aspects of caring. en: Payne S, Ellis-Hill. New perspectives on caring and carers. Oxford: Oxford University Press, 2001, p. 22-43.

2 Heaton J. The gaze and visibility of the carer: a foucauldian analysis of the discourse of informal care. Sociology of Health and Illness 1999; 21: 759-77.

3 Delicado Useros MV, García Fernández MA, López Moreno B, Martínez Sánchez P. Cuidadoras informales: una perspectiva de género. Rev Enfer 2001; (13): 12-6.

4 Celma Vicente M. Cuidadoras informales en el medio hospitalario. Rev Rol Enf 2001; 24(7-8): 503-11.

5 Quero RA. Los cuidados no profesionales en el hospital: la mujer cuidadora. Enfer Clin 2003; 13(6): 348-56.

6 García-Calvente MM, Mateo-Rodríguez I, Maroto-Navarro G. El impacto de cuidar en la salud y la calidad de vida de las mujeres. Gac Sanit 2004; 18(2): 16.

Figura 2. Conceptos en torno al cuidador

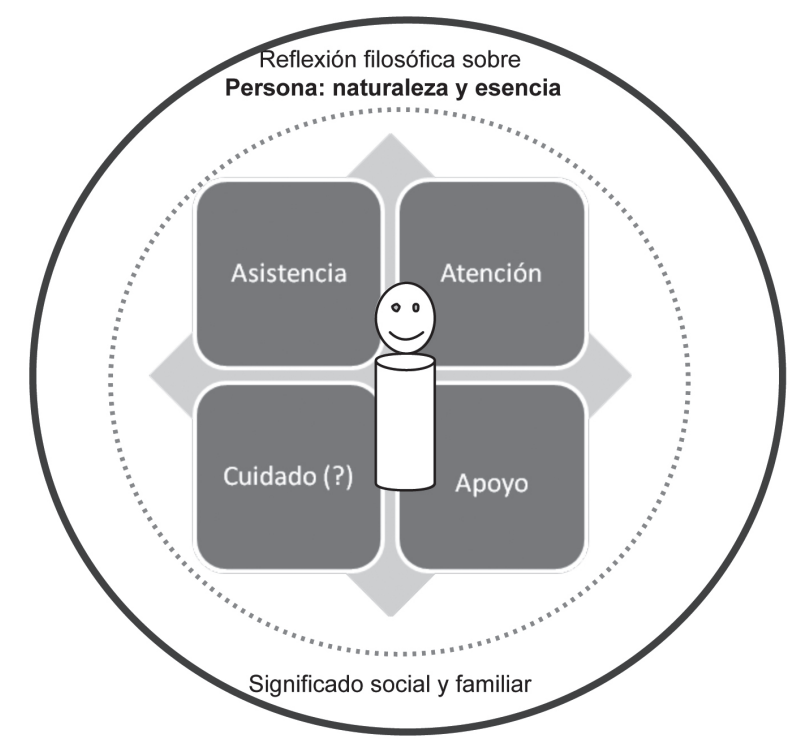


7 Robles Silva L. El cuidado en el hogar a los enfermos crónicos: un sistema de autoatención. Cad. Saúde Pública 2004; 20(2): 618-25.

8 Péculo Carrasco JA, Rodríguez Bouza M, Casal Sánchez MM, Rodríguez Ruíz HJ, Pérez de los Santos OC, Martín Tello JM. Cansancio del cuidador informal en la asistencia sanitaria urgente extrahospitalaria. Rev. Cuidados 2006; 6(1): 15-8.

9 Díaz Álvarez JC. Habilidades de cuidado de los cuidadores familiares de personas en situación de enfermedad crónica. Av Enferm 2007; 25(19): 69-82.

10 Venegas Bustos BC. Habilidad del cuidador y funcionalidad de la persona cuidada. Aquichan 2006; 6(1): 137-47.

11 Ramírez Ordóñez MM. Habilidad del cuidado de los cuidadores principales con cáncer gastrointestinal. Av Enferm 2005; 23(2): 7-17.

12 Pérez Cárdenas C. Repercusión familiar de un diagnóstico oncológico. Rev Psicol [on line]. Disponible: http://pepsic.vsalud.org. Consultada 9 dic. 2010.

13 Cortés Funes F, Abián L, Cortés Funes H. Adaptación psicosocial del paciente oncológico ingresado y del familiar cuidador principal. Psicooncología 2001; 1(1): 83-92.

14 Peón Sánchez MJ. Atención de enfermería hacia el cuidador principal del paciente con enfermedad de alzheimer. Enferm Cient 2004; (3): 264-5.

15 Sánchez B. La experiencia de ser cuidadora de una persona en situación de enfermedad crónica. Investig Educ Enferm 2001; 19(2): 36-49.

16 Escuredo Rodríguez B. El sistema formal de cuidados. Rev Rol Enf 2006; 29(10): 671-6.
17 Morales P, Orfa N. Situación del cuidador familiar de la persona que vive con enfermedad crónica discapacitante en Guatemala. Enf Global 2007; 11: 1-5.

18 Castillo E, Chesla CA, Echeverry G. Satisfacción de los familiares cuidadores con la atención a adultos y niños con cáncer. Colomb Med 2005; 36(Supl 2): 43-8.

19 Muñoz Cobos F, Espinoza Almedro JM, Portillo Strempel J. La familia en la enfermedad terminal (I). Med Fam 2002; 3(3): 190-9.

20 Scherma G.A. How to get organized as a Caregiver. Loss, grief \& Care. 2002; 8(3-4): 127-34.

21 Wai Man Kwan C. Families' experiences of the last office of deceased family members in the hospice setting. International Journal of Palliative Nursing. 2005; 8 (6):26675.

22 Astudillo W, Mendinueta C, Astudillo E. (4ta. ed.). El síndrome del agotamiento en los Cuidados Paliativos. En: Cuidados del enfermo en fase terminal y atención a su familia. EUNSA, Barañain. 2002; 514-25.

23 Marques S, Partezani Rodríguez RA. La familia al cuidado del anciano. Rev Rol Enf 2002; (7-8): 502-10.

24 IV Reunión de Antropología do Mercosur Foro de Investigación: Envejecimiento de la población en el MERCOSUR. Noviembre, 2001. Curitiba. Brasil, Construyendo el concepto cuidador de ancianos. María Cristina de los Reyes.

25 Barrera Ortiz L, Blanco de Camargo L, Figueroa Ingrid P, Pinto Afanador N, Sánchez Herrera B. Habilidad de cuidadores familiares de personas con enfermedad crónica. Mirada internacional. Rev Chía Colombia 2006: 6(6): 22-33.

26 Bergero T., Gómez M, Herrera A, Mayoral F., Grupo de cuidador primario de daño cerebral: una perspectiva de análisis. Rev Asoc Esp Neuropsiq 2000; 20(73): 12735.

27 Vallerand F, Collins-Bohler D, MSN, Templin T, PhD, Hasenau S. Manejo del dolor en pacientes con cáncer que reciben atención domiciliaria. Cáncer Nurs 2007; 30(1): 31-7.

28 Etsuko Maeyama, Masako Kawa, Auki Shirai, Mitsunori Miyashita, Keiko Kazuma, Takeshi Okabe. Experiencias del cuidador familiar en el cuidado de un paciente con cáncer terminal en su casa en Japón. Palliative \& Supportive Care 2007; 5(4): 389-95.

29 Yaquelín Expósito C. La calidad de vida en los cuidadores primarios de pacientes con cáncer. Rev Haban Cienc Méd, [on line]. Disponible: http:// scielo.sld.cu. Consultada 01 noviembre 2010.

30 Brenda Selene B. Alvarado Aguilar S. Desgaste físico y emocional del cuidador primario en cáncer. Rev Cancerología 2009; 4: 39-46.

31 Islas Salas NL, Ramos del Rio B, Aguilar Estrada MG, García Guillen ML. Perfil psicosocial del cuidador primario informal del paciente con EPOC. Rev Inst Nal Enf Resp Mex 2006; 19(4): 266-71.

32 Izquierdo MJ. El cuidado de los individuos y de los grupos: quien se cuida. disponible en www.intercanvis.es/pdf/. Consultada el 10 de oct. del 2009.

33 Diccionario filosófico. [on line]. Disponible en: http://www.filosofia.org/filomat/dfalf.htm. Consultado 6 julio del 2010.

34. Diccionario Soviético de Filosofía. Ed. Pueblos Unidos, Montevideo; 1965, p. 28, disponible [on line] http://www.filosofia.org/enc/ros/ atencion.htm. 